\title{
ON THE COMPUTATION OF IMPASSE POINTS OF QUASI-LINEAR DIFFERENTIAL-ALGEBRAIC EQUATIONS
}

\author{
PATRICK J. RABIER AND WERNER C. RHEINBOLDT
}

\begin{abstract}
We present computational algorithms for the calculation of impasse points and higher-order singularities in quasi-linear differential-algebraic equations. Our method combines a reduction step, transforming the DAE into a singular ODE, with an augmentation procedure inspired by numerical bifurcation theory. Singularities are characterized by the vanishing of a scalar quantity that may be monitored along any trajectory. Two numerical examples with physical relevance are given.
\end{abstract}

\section{INTRODUCTION}

Many applications in science and engineering involve mixed systems of differential and algebraic equations (DAEs). For some examples see, for instance, the monograph [3]. It is hardly surprising that such systems share many properties with ordinary differential equations (ODEs). In fact, recent existence theories $[5,10,11]$ have shown that, in general, a DAE can be reduced locally to an (explicit) ODE on some submanifold of the space of unknown variables.

However, despite the strong analogy between DAEs and ODEs, important differences exist. For instance, from the fact that DAEs are reducible to ODEs only on some submanifold of the solution space it follows that solutions of a DAE can pass only through points on such a submanifold; that is, its initial values must satisfy certain compatibility conditions. Beyond this, solutions of DAEs may exhibit features that solutions of explicit ODEs cannot possess. For instance, the simple problem

$$
x_{1}^{2}+x_{2}=0, \quad \dot{x}_{2}=1, \quad x(0)=(1,-1)
$$

has the unique solution $x(t)=\left((1-t)^{1 / 2}, t-1\right)$, which cannot be continued beyond $t=1$ despite the fact that $x(1)=(0,0)$ and $\lim _{t \rightarrow 1^{-}} x(t)=x(1)$ exist. This situation would be impossible for solutions of explicit ODEs.

In the electrical engineering literature, points where the solutions cease to exist have been called impasse points (see, e.g., [5] or [6], where also other references are given). Although they have no analog in connection with explicit ODEs, these impasse points are closely related to the "singular points" of implicit ODEs. In [9] the most often encountered type of such singularities

Received by the editor August 3, 1992.

1991 Mathematics Subject Classification. Primary 65L05, 65H10, 58F14.

This work was supported in part by ONR-grant N-00014-90-J-1025, NSF-grant CCR-8907654, and AFOSR-grant 900094. 
for implicit ODEs was analyzed, called there standard singular points. In a recent paper [12] it was shown that the geometric reduction theory for DAEs presented in [11] allows for a generalization of the results in [9] to so-called standard impasse points of quasilinear DAEs.

The aim of this article will be to show that the theory of [12] leads naturally to the development of a computational procedure for the explicit computation of impasse points and other singularities of quasilinear DAEs. For this we outline, in $\S \S 2$ and 3 , briefly and without proof, some of the relevant results for singular ODEs and DAEs from the cited earlier papers. Then $\S 4$ presents the details of the computational algorithm and finally, in $\S 5$, we give some numerical examples which show the effectivity of the process.

\section{SiNGULAR POINTS OF ODES}

Definition 2.1. Consider a quasi-linear problem

$$
B(y) \dot{y}=H(y), \quad y(0)=y_{0},
$$

where $B: \mathscr{D} \rightarrow \mathscr{L}\left(\mathbb{R}^{n}\right)$ and $H: \mathscr{D} \rightarrow \mathbb{R}^{n}$ are $C^{1}$ on some open set $\mathscr{D} \subset \mathbb{R}^{n}$. A point $y \in \mathscr{D}$ is a regular point of $(2.1)$ if $\operatorname{rank} B(y)=n$, and a singular point if $\operatorname{rank} B(y)<n$ but $y$ is a limit point of regular points of (2.1).

Clearly, for a regular point $y_{0} \in \mathscr{D}$ the initial value problem (2.1) has a unique solution in a neighborhood of $y_{0}$. But already simple examples show that the behavior of the solutions of (2.1) in a neighborhood of a singular point $y_{0}$ may vary strongly with the type of singularity encountered there. A partial classification of singularities which will be sufficient for our purposes is given next.

Definition 2.2. (i) A singular point $y \in \mathscr{D}$ of the ODE in (2.1) is $r$-singular if

$$
\operatorname{dim} \operatorname{ker} B(y)=r \text {. }
$$

(ii) An $r$-singular point $y$ is called algebraic (resp. geometric) if

$$
H(y) \notin \operatorname{rge} B(y) \quad(\text { resp. } H(y) \in \operatorname{rge} B(y)) .
$$

(iii) An algebraic 1-singular point $y$ is a standard singular point if

$$
D B(y)(u, u) \notin \operatorname{rge} B(y) \quad \forall u \in \operatorname{ker} B(y) \backslash\{0\} .
$$

For an explanation of the terminology "algebraic (resp. geometric) singular point", see [12]. We summarize here briefly the theory developed in [9] for the case of standard singular points (see also [12]). With a standard singular point $y_{0}$ as starting point, the initial value problem $(2.1)$ cannot have a $C^{1}$ solution $y: J \rightarrow \mathscr{D}$ on an open interval $J$ containing the origin. In fact, this would require that $B\left(y_{0}\right) \dot{y}(0)=H\left(y_{0}\right)$, which contradicts (2.3). Thus, at a standard singular point we may expect at best "one-sided" solutions in the following sense.

Definition 2.3. With a standard singular point $y_{0} \in \mathscr{D}$ as starting point, a solution of the initial value problem $(2.1)$ is any continuous function $y: J \rightarrow \mathscr{D}$ defined on an interval $J=[0, T)$ or $J=(-T, 0]$ for some $T>0$ which is of class $C^{1}$ on $J^{0}=J \backslash\{0\}$ and satisfies $y(0)=y_{0}$ and $B(y(t)) \dot{y}(t)=H(y(t))$ for $t \in J^{0}$. 
With

$$
\alpha(y)(u, v)=\left(v^{T} H(y)\right)\left(v^{T} D B(y)(u, u)\right),
$$

the two conditions (2.3) and (2.4) for a standard singular point $y$ are equivalent to

$$
\alpha(y)(u, v) \neq 0 \quad \forall u \in \operatorname{ker} B(y) \backslash\{0\}, \forall v \in \operatorname{ker} B(y)^{T} \backslash\{0\} .
$$

Since (2.5) is a continuous quadratic form in $u$ and in $v$, its value must be either positive or negative for all pairs of nonzero vectors $u \in \operatorname{ker} B(y), v \in$ $\operatorname{ker} B(y)^{T}$ if only this holds for one such pair of vectors.

The principal existence result for solutions near standard singular points can now be phrased as follows (see [9, Theorem 5.1]).

Theorem 2.1. Let $y_{0} \in \mathscr{D}$ be a standard singular point of the $O D E$ in (2.1). Then the initial value problem (2.1) has exactly two solutions which are both defined on $J=[0, T)$ or on $J=(-T, 0]$ for some $T>0$ depending upon $\alpha\left(y_{0}\right)(u, v)>0$ or $\alpha\left(y_{0}\right)(u, v)<0$, respectively, for some pair of nonzero vectors $(u, v) \in \operatorname{ker} B(y) \times \operatorname{ker} B(y)^{T}$. Moreover, $\|\dot{y}(t)\|$ tends to infinity as $t \in J \backslash\{0\}$ tends to zero.

Theorem 2.1 implies that a solution of (2.1) starting at some regular point can reach a standard singular point $y_{0}$ at some later time only if the form (2.5) is negative at $y_{0}$. Standard singular points $y_{0}$ with positive form $\alpha\left(y_{0}\right)$ obviously can never be reached in increasing time. Thus, in view of the theorem, the following notation is appropriate.

Definition 2.4. A standard singular point $y_{0}$ of $(2.1)$ is accessible or inaccessible if $\alpha\left(y_{0}\right)(u, v)<0$ or $\alpha\left(y_{0}\right)(u, v)>0$, respectively, for some pair of nonzero vectors $(u, v) \in \operatorname{ker} B(y) \times \operatorname{ker} B(y)^{T}$.

The theorem asserts that accessible standard singular points are reached in finite time by trajectories emanating elsewhere in $\mathscr{D}$. Since these trajectories cannot be continuously extended beyond these points, they represent "catastrophes" for the solutions of (2.1), and standard ODE-solvers fail near such points. It can also be shown (loc. cit.) that no small perturbation of the initial condition (and/or of $B$ or $H$ ) can affect the eventual encounter of such points.

Standard singular points are analogous to limit points of parametrized nonlinear equations

$$
F(z, \lambda)=0 .
$$

Suppose, indeed, that $F: \mathscr{D} \rightarrow \mathbb{R}^{n}$ is of class $C^{1}$ on some open set $\mathscr{D} \subset$ $\mathbb{R}^{n} \times \mathbb{R}^{1}$ and that $z: J \rightarrow \mathbb{R}^{n}$ is a $C^{1}$ mapping on an open interval $J$ such that $(z(\lambda), \lambda) \in \mathscr{D}$ and $F(z(\lambda), \lambda)=0$ for $\lambda \in J$. Then

$$
\left(\begin{array}{cc}
D_{z} F(z(\lambda), \lambda) & 0 \\
0 & 1
\end{array}\right)\left(\begin{array}{c}
z^{\prime}(\lambda) \\
\lambda^{\prime}
\end{array}\right)=\left(\begin{array}{c}
-D_{\lambda} F(z(\lambda), \lambda) \\
1
\end{array}\right),
$$

where primes indicate differentiation with respect to $\lambda$, and hence $(2.7)$ is an ODE of the quasi-linear form (2.1). The singular points of (2.7) are exactly those $(z, \lambda) \in \mathscr{D}$, for which $\operatorname{rank} D_{z} F(z, \lambda)<n$, that is, which are foldpoints of $F$ with respect to $\lambda$ (see, e.g., [13]). The simplest foldpoints are the limit points, for which $\operatorname{dim} \operatorname{ker} D_{z} F(z, \lambda)=1$ and $D_{\lambda} F(z, \lambda) \notin \operatorname{rge} D_{z} F(z, \lambda)$. Obviously, these two properties correspond to the conditions (2.2) and (2.3) 
characterizing algebraic 1-singular points, and it is readily checked that (2.4) holds exactly for the simple limit points of $F$ (with respect to $\lambda$ ); see, e.g., [13].

Foldpoints of a parametrized nonlinear system are typically computed by solving a suitably augmented form of the system. It is natural to consider the same approach for the computation of singular points of ODEs.

Let $y=y(t)$ be a $C^{1}$ solution of (2.1) and suppose that a $C^{1}$ function $\tau: \mathbb{R}^{1} \rightarrow \mathbb{R}^{1}$ with strictly positive derivative is used to define a transformation $t=\tau(s)$ of the independent variable $t$. Then $\eta(s)=y(\tau(s))$ satisfies

$$
B(\eta) \frac{d \eta}{d s}=\frac{d \tau}{d s} H(\eta)
$$

By Theorem 2.1 the derivative $d y / d t$ becomes infinite when the solution approaches a standard singular point $y^{*}$. This suggest that $\tau$ should be chosen such that $d \tau / d s$ tends to zero as we approach $y^{*}$ but $d \eta / d s$ remains bounded. For instance, we may wish to specify $\tau$ implicitly by using a normalization $c^{T}(d \eta / d s)=1$ with a suitable vector $c \in \mathbb{R}^{n}$.

This normalization may be obtained by means of an augmented system of the form

$$
\left(\begin{array}{cc}
B(y) & -H(y) \\
c^{T} & 0
\end{array}\right)\left(\begin{array}{l}
v(y) \\
\gamma(y)
\end{array}\right)=\left(\begin{array}{l}
0 \\
1
\end{array}\right)
$$

where $c \in \mathbb{R}^{n}$ is chosen such that at some "current" point $y_{c} \in \mathscr{D}$ under consideration the matrix of (2.8) is nonsingular. Certainly, such an augmentation can be found if and only if $y_{c}$ is either a regular point of (2.1) or an algebraic 1 -singular point.

Hence the matrix of (2.8) remains nonsingular for all $y$ in some open neighborhood $\mathscr{U}_{c} \subset \mathscr{D}$ of $y_{c}$, whence for fixed $y \in \mathscr{U}_{c}$ the solution $(v(y), \gamma(y)) \in$ $\mathbb{R}^{n+1}$ of (2.8) is unique. Obviously, we have $v(y) \neq 0$ for all $y \in \mathscr{U}_{c}$ and $\gamma(y) \neq 0$ for all regular points $y \in \mathscr{U}_{c}$ of (2.1). Moreover, because of (2.3) we see that $\gamma\left(y^{*}\right)=0$ at any algebraic 1 -singular point $y^{*} \in \mathscr{U}_{c}$.

For any regular point $y_{0} \in \mathscr{U}_{c}$ the initial value problem $(2.1)$ has a unique, local $C^{1}$ solution $y:[0, T) \rightarrow \mathscr{U}_{c}$ for some $T>0$ such that $B(y(t))$ is nonsingular for $t \in[0, T$ ) (we confine attention to positive time since we assume that the system evolves from $t=0$ on). Suppose that $\gamma\left(y_{0}\right)>0$. Then $\gamma(y(t))>0$ for $0 \leq t<T$ and the initial value problem

$$
\frac{d t}{d s}=\gamma(y(t)), \quad s \geq 0, \quad t(0)=0,
$$

has a unique, monotonically increasing solution $\tau:[0, \sigma) \rightarrow \mathbb{R}^{1}$ with $0<$ $\tau(\sigma)<T$. Hence, as desired, $\tau$ defines a transformation of the independent variable $t$ of (2.1). As before, we set

$$
\eta(s)=y(\tau(s)), \quad s \in[0, \sigma), \quad \eta(0)=y_{0} .
$$

Together with $(2.9)$, the chain rule provides that

$$
\frac{d \eta}{d s}(s)=\gamma(\eta(s)) \frac{d y}{d t}(\tau(s)), \quad s \in[0, \sigma),
$$

whence by (2.1) and (2.8) it follows that

$$
B(y(t))\left[\gamma(y(t)) \frac{d y}{d t}(t)-v(y(t))\right]=0, \quad 0 \leq t<T,
$$


and therefore, by $(2.10),(2.11)$, and the invertibility of $B(y(t))$, that

$$
\frac{d \eta}{d s}(s)=v(\eta(s)), \quad \eta(0)=y_{0}, \quad s \in[0, \sigma) .
$$

Proposition 2.1. Suppose that the solution $y=y(t)$ of (2.1) tends to some standard singular point $y^{*} \in \mathscr{U}_{c}$ and hence has been extended to a maximal interval $\left[0, T^{*}\right)$ such that $\lim _{t \rightarrow T^{*}} y(t)=y^{*}$. (Necessarily, $y^{*}$ is an algebraic 1 -singular point since the matrix of the augmentation (2.8) is invertible at $y^{*}$ by hypothesis). Then

(i) There exists $\sigma^{*}<\infty$ such that the solution $t=\tau(s)$ of (2.9) is defined for $s \in\left[0, \sigma^{*}\right)$ and that $\lim _{s \rightarrow \sigma^{*}} \tau(s)=T^{*}$.

(ii) The solution of (2.12) is defined and of class $C^{1}$ on $\left[0, \sigma^{*}+\epsilon\right)$ for some $\epsilon>0$.

(iii) If, in addition, $y^{*}$ is a standard singular point, then $\gamma(\eta(s))$ changes sign as $s$ crosses $\sigma^{*}$.

Proof. Let $J$ denote the set of all $\bar{\sigma}$ such that there exists a $C^{1}$ solution of (2.9) for $s \in[0, \bar{\sigma})$ satisfying $0 \leq \tau(s)<T^{*}$ on that interval. Clearly, $J$ is not empty and hence $\sigma^{*}=\sup \{\bar{\sigma}: \bar{\sigma} \in J\}$ is well defined. Thus, there exists a $C^{1}$ solution of (2.9) for $s \in\left[0, \sigma^{*}\right)$ satisfying $0 \leq \tau(s)<T^{*}$ on this interval. In order to show that $\lim _{s \rightarrow \sigma^{*}} \tau(s)=T^{*}$, note that $\tau$ remains monotonically increasing on $\left[0, \sigma^{*}\right)$ and hence that $\lim _{s \rightarrow \sigma^{*}} \tau(s)=\tau^{*} \leq T^{*}$ exists. Suppose that $\tau^{*}<T^{*}$. Then, by the continuity of $\gamma$, the compactness of $y\left(\left[0, \tau^{*}\right]\right)$, and the fact that $\gamma(y(t))>0$ for $t \in\left[0, T^{*}\right)$, it follows that there exists a positive constant $\gamma_{0}$ for which $\gamma(y(t)) \geq \gamma_{0}$ in $\left[0, \tau^{*}\right]$. This implies that $\sigma^{*}<\infty$, for otherwise $\tau(s) \geq \gamma_{0} s$ for $s \in[0, \infty)$ and hence $\lim _{s \rightarrow \infty} \tau(s)=\infty$ in contradiction with $0 \leq \tau(s) \leq \tau^{*}<\infty$ for $s \in\left[0, \sigma^{*}\right)=[0, \infty)$. But now, by setting $\tau\left(\sigma^{*}\right)=\tau^{*}$, we can define a continuous extension of $\tau$ to some interval $\left[0, \sigma^{*}+\epsilon\right]$ with sufficiently small $\epsilon>0$ such that $0 \leq \tau(s)<T^{*}$. Thus, assuming $\tau^{*}<T^{*}$, we obtain a contradiction with the maximality of $\sigma^{*}$. This shows that $\tau^{*}=T^{*}$.

The above arguments show only that $\sigma^{*} \leq \infty$, but we now prove that $\sigma^{*}<\infty$. In fact, assume, to the contrary, that $\sigma^{*}=\infty$, so that $\eta$ is defined in $[0, \infty)$. Since, by construction, $c^{T} v(\eta(s)) \equiv 1$, it follows from (2.12) that $c^{T}(d \eta / d s)(s) \equiv 1$, whence $c^{T} \eta(s)=c^{T} y_{0}+s$. This implies that $\lim _{s \rightarrow \infty} c^{T} \eta(s)$ $=\infty$ and hence also that $\lim _{s \rightarrow \infty}\|\eta(s)\|=\infty$. But then we arrive at a contradiction since $\|\eta(s)\|=\|y(\tau(s))\|$ and $\|y(t)\|$ is bounded on the compact interval $\left[0, T^{*}\right]$. This proves $(\mathrm{i})$.

It follows from (i) that (2.12) has a unique solution $\eta$ for $s \in\left[0, \sigma^{*}\right)$, and $\eta\left(\sigma^{*}\right)=y^{*}$ defines a continuous extension of $\eta$. Therefore, the solution of (2.12) can be extended to a larger interval $\left[0, \sigma^{*}+\epsilon\right)$ with some $\epsilon>0$ as claimed in (ii).

As noted earlier, we have $\gamma(\eta(s))>0$ for $0 \leq s<\sigma^{*}$ and $\gamma\left(\eta\left(\sigma^{*}\right)\right)=$ $\gamma\left(y^{*}\right)=0$. Thus, $\sigma^{*}$ is the first zero of $\gamma(\eta(s))$ in $\left[0, \sigma^{*}+\epsilon\right)$. If now $y^{*}$ is a standard singular point, $\gamma(\eta(s))$ must change sign as $s$ crosses $\sigma^{*}$. For this, note that by differentiation of

$$
B(y) v(y)=\gamma(y) H(y), \quad y \in \mathscr{U}_{c},
$$

together with $\gamma\left(y^{*}\right)=0$, we obtain for all $h \in \mathbb{R}^{n}$ that

$$
D B\left(y^{*}\right)\left(h, v\left(y^{*}\right)\right)+B\left(y^{*}\right) D v\left(y^{*}\right) h=\left(D \gamma\left(y^{*}\right) h\right) H\left(y^{*}\right) .
$$


For $h=v\left(y^{*}\right) \neq 0$ and any nonzero vector $w \in \operatorname{ker} B\left(y^{*}\right)^{T}$ it follows from (2.5) and (2.6) that

$$
D \gamma\left(y^{*}\right) v\left(y^{*}\right)=\frac{w^{T} D B\left(y^{*}\right)\left(v\left(y^{*}\right), v\left(y^{*}\right)\right)}{w^{T} H\left(y^{*}\right)}=\frac{\alpha\left(y^{*}\right)\left(v\left(y^{*}\right), w\right)}{\left(w^{T} H\left(y^{*}\right)\right)^{2}} \neq 0 .
$$

Since $(d(\gamma \circ \eta) / d s)\left(\sigma^{*}\right)=D \gamma\left(y^{*}\right) v\left(y^{*}\right)$, this proves that indeed $(\gamma \circ \eta)(s)$ must change sign as $s$ crosses $\sigma^{*}$.

Altogether, therefore, by solving (2.12) and monitoring the first sign change of $\gamma(\eta(s))$, we can calculate $\sigma^{*}$ and hence $y^{*}=\eta\left(\sigma^{*}\right)$. The value of $T^{*}$ is then given by

$$
T^{*}=\int_{0}^{\sigma^{*}} \gamma(\eta(s)) d s
$$

which follows directly from (2.9) and $\lim _{s \rightarrow \sigma^{*}} \tau(s)=T^{*}$.

The augmentation procedure described here is designed to work in the neighborhood of a standard singular point. But in practice, also higher-order singularities $y^{*} \in \mathscr{D}$ are encountered, where the matrix of the simple augmented system (2.8) becomes singular. In order to avoid difficulties near such points, we may work with an overdetermined augmented system of the form

$$
\left(\begin{array}{ccc}
B(y) & -H(y) & E \\
C^{T} & 0 & 0
\end{array}\right)\left(\begin{array}{c}
V(y) \\
w(y)^{T} \\
Z(y)^{T}
\end{array}\right)=\left(\begin{array}{c}
0_{n \times q} \\
I_{q}
\end{array}\right)
$$

Here, for given $q, 1<q \leq n$, the matrices $E$ and $C$ have dimension $n \times(q-1)$ and $n \times q$, respectively, and, correspondingly, in the solution, $V(y), w(y)$, and $Z(y)$ are blocks of size $n \times q, q \times 1$, and $q \times(q-1)$, respectively. As before, the matrices $E$ and $C$ are chosen such that at some "current" point $y_{c} \in \mathscr{D}$ the matrix of (2.14) is nonsingular and hence remains nonsingular for all $y$ in some open neighborhood $\mathscr{U}_{c} \subset \mathscr{D}$ of $y_{c}$. Thus, for each $y \in \mathscr{U}_{c}$ the solution of (2.14) is unique. Clearly, for sufficiently large $q$ a suitable choice of $E$ and $C$ exists irrespective of $\operatorname{dim} \operatorname{ker} B\left(y_{c}\right)$, and even in the case when $y_{c}$ is a geometric singularity, i.e., $H\left(y_{c}\right) \in \operatorname{rge} B\left(y_{c}\right)$ (unless $H\left(y_{c}\right)=0$; but see Remark 2.1 further below).

We summarize some basic properties of the augmentation (2.14):

Proposition 2.2. For given $q>1$ and $y \in \mathscr{U}_{c}$ the solution of (2.14) satisfies

$$
\operatorname{dim} \operatorname{ker} B(y)=\operatorname{dim} \operatorname{ker}(w(y), Z(y)),
$$

and $\operatorname{rank} Z(y)=q-1$ implies that $\operatorname{rank}(B(y),-H(y))=n$. The converse holds if $y$ is a regular point or if $H(y) \notin \operatorname{rge} B(y)$ (and hence $y$ is an algebraic 1-singular point).

Proof. Generally, for $y \in \mathscr{U}_{c}$ we have

$$
B(y) V(y)=(H(y),-E)(w(y), Z(y))^{T},
$$

as well as $\operatorname{rank} V(y)=q$ (since $\left.C^{T} V(y)=I_{q}\right)$ and $\operatorname{rank}(H(y),-E)=q$ ( since the matrix of (2.14) is invertible), which together imply the first assertion. Indeed, since both $V(y)$ and $(H(y),-E)$ are $n \times q, q \leq n$, and have maximum rank $q$, we infer from (2.16) that

$$
\text { dim } \operatorname{ker} B(y) V(y)=\operatorname{dim} \operatorname{ker}(w(y), Z(y))^{T}=\operatorname{dim} \operatorname{ker}(w(y), Z(y))
$$


(recall that $(w(y), Z(y))$ is $q \times q)$. Moreover, using again the fact that $\operatorname{ker} V(y)$ $=\{0\}$, we see that

$$
\operatorname{dim} \operatorname{ker} B(y) V(y) \leq \operatorname{dim} \operatorname{ker} B(y) .
$$

Thus, to complete the proof of (2.15), it suffices to show that the converse inequality of (2.17) holds. This follows at once if we can show that $\operatorname{ker} B(y) \subset$ $\operatorname{rge} V(y)$. For this, let $u \in \operatorname{ker} B(y)$, so that

$$
\left(\begin{array}{ccc}
B(y) & -H(y) & E \\
C^{T} & 0 & 0
\end{array}\right)\left(\begin{array}{c}
u \\
0_{1 \times 1} \\
0_{(q-1) \times 1}
\end{array}\right)=\left(\begin{array}{l}
0_{n \times 1} \\
C^{T} u
\end{array}\right) .
$$

On the other hand, multiplying both sides of $(2.14)$ by $C^{T} u$, we get

$$
\left(\begin{array}{ccc}
B(y) & -H(y) & E \\
C^{T} & 0 & 0
\end{array}\right)\left(\begin{array}{c}
V(y) C^{T} u \\
w(y)^{T} C^{T} u \\
Z(y)^{T} C^{T} u
\end{array}\right)=\left(\begin{array}{c}
0_{n \times 1} \\
C^{T} u
\end{array}\right) .
$$

But the systems (2.18) and (2.19) have the same right-hand side, and hence, because the matrix is invertible, the solutions are identical whence, in particular, $u=V(y) C^{T} u$ and therefore $u \in \operatorname{rge} V(y)$.

If $\operatorname{rank} Z(y)=q-1$, then $\operatorname{rge} E Z^{T}=\operatorname{rge} E$. Therefore, $\operatorname{rge} E \subset$ $\operatorname{rge}(B(y),-H(y))$ and hence $\operatorname{rge}(B(y),-H(y), E)=\operatorname{rge}(B(y),-H(y))$, which for $\operatorname{rank}(B(y),-H(y))<n$ contradicts the nonsingularity of the matrix of (2.14). Conversely, that rank $Z(y)=q-1$ if $y$ is regular is obvious from (2.15).

To prove that $\operatorname{rank}(B(y),-H(y))=n$ and $H(y) \notin \operatorname{rge} B(y)$ imply $\operatorname{rank} Z(y)=q-1$, suppose that $H(y) \notin \operatorname{rge} B(y)$ and $\operatorname{rank} Z(y) \leq q-2$, so that $\operatorname{dim} \operatorname{ker} Z(y)^{T} \geq 2$. Let $u_{\alpha}, \alpha=1,2$, be two linearly independent vectors in $\operatorname{ker} Z(y)^{T}$. By (2.16) we have

$$
B(y) V(y) u_{\alpha}=\left(w(y)^{T} u_{\alpha}\right) H(y), \quad \alpha=1,2,
$$

and hence $w(y)^{T} u_{\alpha}=0, \alpha=1,2$, since $H(y) \notin \operatorname{rge} B(y)$. Thus, $u_{\alpha} \in$ $\operatorname{ker}(w(y), Z(y))^{T}, \alpha=1,2$. By $(2.15)$ the linear independence of the two vectors implies that $\operatorname{dim} \operatorname{ker} B(y) \geq 2$, which in turn implies that $\operatorname{rank}(B(y),-H(y))$ $<n$.

For $y \in \mathscr{U}_{c}$ and any vector $a(y) \in \operatorname{ker} Z(y)^{T}$ we have

$$
B(y) v(y)=\gamma(y) H(y), \quad c(y)^{T} v(y)=a(y)^{T} a(y),
$$

with

$$
v(y)=V(y) a(y), \quad c(y)=C a(y), \quad \gamma(y)=w(y)^{T} a(y) .
$$

This has the general form of (2.8), and as before, $\gamma(y)=0$ implies that $B(y)$ is singular if $a(y) \neq 0$. But, the converse is true only if $H(y) \notin \operatorname{rge} B(y)$. Moreover, in order to produce smooth functions $v, c$, and $\gamma$, the vector $a(y)$ must depend smoothly on $y$. This is easily guaranteed as long as $\operatorname{rank} Z(y)=$ $q-1$ but not in general unless we drop the assumption that $a(y) \neq 0$. Let $Z^{i}(y)$ denote the $(q-1) \times(q-1)$ submatrix obtained from $Z(y)$ by deleting the $i$ th column. Then the vector

$$
\begin{array}{r}
a(y)=\left(a_{1}(y), \ldots, a_{q}(y)\right)^{T}, \quad a_{i}(y)=(-1)^{i} \operatorname{det} Z^{i}(y), \\
i=1, \ldots, q,
\end{array}
$$


obviously depends smoothly on $y$ and satisfies $Z(y)^{T} a(y)=0$ (see, e.g., [16, Appendix II]). Moreover, we have $a(y) \neq 0$ exactly if $\operatorname{rank} Z(y)=q-1$.

For this choice of $a(y)$ the following result holds.

Proposition 2.3. For $y \in \mathscr{U}_{c}$ and with the vectors (2.21) consider the relations (2.20). Then $\gamma(y)=0$ exactly if $B(y)$ is singular.

Proof. If $\gamma(y)=0$, then either $\operatorname{rank} Z(y)<q-1$, in which case, by (2.15), $B(y)$ is singular, or $\operatorname{rank} Z(y)=q-1$, whence $a(y) \neq 0$ and thus also $v(y) \neq$ 0 , which together with $B(y) v(y)=0$ implies again that $B(y)$ is singular. Conversely, suppose that $B(y)$ is singular. In the case $\operatorname{rank} Z(y)=q-1$, we have again $v(y) \neq 0$, and Proposition 2.2 ensures that $H(y) \notin \operatorname{rge} B(y)$. Thus, the first equation (2.20a) leads to a contradiction unless $\gamma(y)=0$. On the other hand, for $\operatorname{rank} Z(y)<q-1$ we necessarily have $\gamma(y)=0$ because $a(y)=0$ in that case.

By Proposition 2.2 we see that when $y$ is a geometric singular point, then necessarily $\operatorname{rank} Z(y)<q-1$. Such points constitute "higher" singularities. Our choice (2.21) of $a(y)$ evidently transforms these points into equilibrium points of the dynamic system (2.12) with $v$ as in (2.20b).

Computationally, the simplest case arises with $q=2$, where $(2.14)$ has the form

$$
\left(\begin{array}{ccc}
B(y) & -H(y) & e \\
c_{1}^{T} & 0 & 0 \\
c_{2}^{T} & 0 & 0
\end{array}\right)\left(\begin{array}{cc}
v_{1}(y) & v_{2}(y) \\
w_{1}(y) & w_{2}(y) \\
z_{1}(y) & z_{2}(y)
\end{array}\right)=\left(\begin{array}{ll}
0 & 0 \\
1 & 0 \\
0 & 1
\end{array}\right)
$$

Hence, the vector $(2.21)$ becomes here $a(y)=\left(-z_{2}(y), z_{1}(y)\right)$, and we obtain

$$
\begin{aligned}
& v(y)=z_{2}(y) v_{1}(y)-z_{1}(y) v_{2}(y), \\
& \gamma(y)=z_{2}(y) w_{1}(y)-z_{1}(y) w_{2}(y), \\
& c(y)=z_{2}(y) c_{1}(y)-z_{1}(y) c_{2}(y) .
\end{aligned}
$$

Proposition 2.3 implies that $\gamma(y)$ does not vanish on a trajectory terminating at a singular point $y^{*} \in \mathscr{U}_{c}$ unless $y=y^{*}$. Obviously, (2.22) has the same general form as the simple augmentation (2.8), and the computational procedure is the same as before, namely, we form and solve the explicit equation (2.12). The only difference is that the normalization condition now involves the nonconstant vector $c(y)$ while in (2.8) this vector was constant.

The constancy of $c$ was used in Proposition 2.1 to prove that for an algebraic 1-singular point there exists $\sigma^{*}<\infty$ such that the solution $t=\tau(s)$ of (2.9) is defined in $\left[0, \sigma^{*}\right)$ and that $\lim _{s \rightarrow \sigma^{*}} \tau(s)=T^{*}$. The result is easily extended to the case when $c$ depends on $y$. For this, note that the proof of the existence of $\sigma^{*} \leq \infty$ carries over verbatim and irrespective of the singularity encountered at $y^{*}$; all that is needed is that the matrix $(2.22)$ be invertible at $y^{*}$. Now if $y^{*}$ is an algebraic 1 -singular point and $\sigma^{*}=\infty$, we conclude from $c(\eta(s))^{T} v(\eta(s))=\|a(y)\|_{2}^{2}$ that $c\left(y^{*}\right)^{T} v(\eta(s)) \geq \epsilon>0$ for all $s$ close to $\sigma^{*}$. Indeed, rank $Z\left(y^{*}\right)=q-1$ by Proposition 2.2 and hence $a\left(y^{*}\right) \neq 0$. In other words, for sufficiently large $s$ it follows that $c\left(y^{*}\right)^{T}(d \eta / d s)(s) \geq \epsilon$ and therefore that $c\left(y^{*}\right)^{T} \eta(s) \geq \epsilon\left(s-s_{0}\right)+c\left(y^{*}\right)^{T} \eta\left(s_{0}\right)$ for $s \geq s_{0}$ and $s_{0}$ large enough. As before, this contradicts the boundednes of $\|\eta(s)\|$ for $s \geq 0$. 
If $y^{*}$ is a standard singular point, then $\gamma(\eta(s))$ changes sign as $s$ crosses $\sigma^{*}$. On the other hand, if $y^{*}$ is not an algebraic 1 -singular point, then we can no longer ascertain that $\sigma^{*}<\infty$.

Remark 2.1. The matrix of any augmentation (2.14) and, in particular, that of (2.8) or (2.22), will be singular at any point $y \in \mathscr{U}_{c}$ where $H(y)=0$, that is, at any stationary (equilibrium) point of the autonomous ODE (2.1). This reflects the fact that any regular stationary point can be reached only in infinite time, and hence our scale transformation must become undetermined along such trajectories. Clearly, the natural resolution of this difficulty is to make the system (2.1) nonautonomous by adding the equation $\dot{t}=1$.

\section{Singular POINTS OF DAEs}

In this section we turn to differential-algebraic systems of the following form.

Definition 3.1. The equation

$$
A(x) \dot{x}=G(x),
$$

with $C^{2}$ mappings $A: \mathscr{D} \rightarrow \mathscr{L}\left(\mathbb{R}^{n}\right)$ and $G: \mathscr{D} \rightarrow \mathbb{R}^{n}$ on some open set $\mathscr{D} \subset$ $\mathbb{R}^{n}$, is a quasi-linear DAE on $\mathscr{D}$ if

$$
G(x) \in \operatorname{rge} A(x), x \in \mathscr{D} \Longrightarrow \operatorname{rank} A(x)=r<n
$$

and if the mapping

$$
(x, p) \in \mathscr{D} \times \mathbb{R}^{n} \longmapsto A(x) p-G(x) \in \mathbb{R}^{n}
$$

is a submersion.

The submersion property of (3.3) requires that for every $(x, p) \in \mathscr{D} \times \mathbb{R}^{n}$ the mapping

$$
(h, k) \in \mathbb{R}^{n} \times \mathbb{R}^{n} \longmapsto(D A(x) h) p+A(x) k-D G(x) h \in \mathbb{R}^{n}
$$

is onto (see, e.g., [1]). As a consequence, the set

$$
M=\left\{(x, p) \in \mathscr{D} \times \mathbb{R}^{n}: A(x) p-G(x)=0\right\}
$$

is a closed $n$-dimensional $C^{2}$ submanifold of $\mathscr{D} \times \mathbb{R}^{n}$.

In [11] a geometric procedure was developed for reducing an implicit DAE, $F(x, \dot{x})=0$, to an ODE on a manifold locally near a point $\left(x^{0}, p^{0}\right) \in F^{-1}(0)$. A simplified version of this reduction process for quasi-linear DAEs (3.1) is given in [12]. In that case the reduction is local only in the first variable, owing to the linearity of the equation in $\dot{x}$. We summarize briefly this process for (3.1).

Set

$$
W=\{x \in \mathscr{D}: G(x) \in \operatorname{rge} A(x)\},
$$

so that $(x, p) \in M$ if and only if $x \in W$ and hence $W=\pi(M)$, where $\pi: \mathscr{D} \times \mathbb{R}^{n} \rightarrow \mathscr{D}$ is the projection onto the first factor. Under the conditions of Definition 3.1 it can be shown (see [12, Proposition 3.1]) that $W$ is an $r$ dimensional $C^{2}$ submanifold of $\mathscr{D}$ and that $W$ is closed in $\mathscr{D}$ if the set $\{x \in \mathscr{D}: \operatorname{rank} A(x)=r\}$ is closed in $\mathscr{D}$.

For any $C^{1}$ solution $x: J \rightarrow \mathscr{D}$ of (3.1) on an open interval $J \subset \mathbb{R}^{1}$ we must have $x(t) \in W$ for $t \in J$ and thus $(x(t), \dot{x}(t)) \in T W$ for all $t \in J$, 
where we view here the tangent bundle $T W$ as a subset of $T \mathbb{R}^{n}=\mathbb{R}^{n} \times \mathbb{R}^{n}$. Hence, $(x(t), \dot{x}(t)) \in M$ implies that $(x(t), \dot{x}(t)) \in T W \cap M$ for all $t \in J$. The desired reduction of (3.1) now requires a (local) characterization of $T W \cap M$.

For this, let $x^{0} \in W$. Then there exist open subsets $\mathscr{V} \subset W$ and $\mathscr{U} \subset \mathbb{R}^{r}$ and a $C^{2}$ map $\varphi: \mathscr{U} \rightarrow \mathbb{R}^{n}$ which is a diffeomorphism of $\mathscr{U}$ onto $\mathscr{V}$. In other words, $\varphi^{-1}$ is a chart of $W$ at $x^{0}$.

Evidently, $\mathscr{U}$ and $\mathscr{V}$ may be chosen small enough such that there is a linear subspace $Z^{0} \subset \mathbb{R}^{n}$ which complements $\operatorname{rge} A(\varphi(y))$ for all $y \in \mathscr{U}$. Let $P^{0}$ be the projection of $\mathbb{R}^{n}$ onto $\operatorname{rge} A\left(x^{0}\right)$ along $Z^{0}$, and $L^{0}$ any linear isomorphism from $\operatorname{rge} A\left(x^{0}\right)$ onto $\mathbb{R}^{r}$. Then $L^{0} P^{0}$ is a linear isomorphism of $\operatorname{rge} A(\varphi(y))$ onto $\mathbb{R}^{r}$ for all $y \in \mathscr{U}$, and it follows that

$$
\{(x, p) \in T W \cap M, x \in \mathscr{V}\} \Leftrightarrow\left\{\begin{array}{l}
x=\varphi(y), p=D \varphi(y) q, \\
B(y) q-H(y)=0,
\end{array}\right.
$$

where we have set

$$
B(y)=L^{0} P^{0} A(\varphi(y)) D \varphi(y), \quad H(y)=L^{0} P^{0} G(\varphi(y)) .
$$

It is plain that the operators $B$ and $H$ map into $\mathscr{L}\left(\mathbb{R}^{r}\right)$ and $\mathbb{R}^{r}$, respectively, and are of class $C^{1}$.

If the interval of definition $J \subset \mathbb{R}^{1}$ of the solution is restricted to ensure that $x(J) \subset \mathscr{V}$ and therefore that $(x(t), \dot{x}(t)) \in T W \cap M$ and $x(t) \in \mathscr{V}$ for all $t \in J$, then the $C^{1}$ function

$$
y: J \rightarrow \mathscr{U}, \quad y(t)=\varphi^{-1} \circ x(t),
$$

is a $C^{1}$ solution of the equation

$$
B(y) \dot{y}=H(y),
$$

called the reduction of $(3.1)$ near $x^{0}$. Conversely, for any $C^{1}$ solution $y: J \rightarrow$ $\mathscr{U}$ of (3.9) the function $x(t)=\varphi \circ y(t)$ is a $C^{1}$ solution of (3.1).

Evidently, if $B(y)$ in (3.9) satisfies the conditions of Definition 2.1, then the augmentation procedure of $\S 2$ can be applied. This is the case when (3.1) has index 1 in the sense of the following definition.

Definition 3.2. The quasi-linear system (3.1) is a geometrically nonsingular DAE of index 1 if

$$
\left\{x \in W, G(x) \in \operatorname{rge} A(x)_{\left.\right|_{T_{x} W}}\right\} \Rightarrow \operatorname{rank} A(x)_{\mid T_{x} W}=\operatorname{rank} A(x)(=r) .
$$

Remark 3.1. A geometrically nonsingular DAE of index 1 may reduce to a singular ODE with algebraic singularities in the vicinity of some point. In particular, the impasse points discussed below are of this type. On the other hand, if (3.10) does not hold, there are several possibilities:

(a) $\left\{x \in W, G(x) \in \operatorname{rge} A(x)_{\left.\right|_{T_{x} W}}\right\}$ implies $\operatorname{rank} A(x)_{\left.\right|_{T_{x} W}}=\rho<r$. If so, the reduction (3.9) of (3.1) is a DAE and not an ODE as in the index-1 case. This leads to higher-index DAEs as defined in [11].

(b) $\operatorname{rank} A(x)_{\left.\right|_{x} w}=r$ for "most" but not all $x \in W$ satisfying $G(x) \in$ rge $A(x)_{\left.\right|_{T_{x}} w}$. If so, the reduction (3.9) of (3.1) is a singular ODE with geometric singularities. We shall encounter such a situation in our examples later, and it seems appropriate to call (3.1) a geometrically singular DAE with index 1 . 
(c) rank $A(x)_{\left.\right|_{T_{x} W}}$ is neither constant nor mostly equal to $r$. If so, the reduction (3.9) of (3.1) is not a DAE in the sense of Definition 3.1, nor a singular ODE. No theory exists for this case, which fortunately does not seem to occur too frequently in the applications.

From (3.10) it follows that for $x^{0} \in W_{1} \equiv \pi(T W \cap M) \subset W$ we have $B\left(y^{0}\right) \in$ $\mathrm{GL}\left(\mathbb{R}^{r}\right)$, where $y^{0}=\varphi^{-1}\left(x^{0}\right)$, and this provides the basis of the following existence and uniqueness theorem for (3.1).

Theorem 3.1. Let (3.1) be a geometrically nonsingular DAE of index 1. Then, for any $x^{0} \in W_{1}=\pi(T W \cap M) \subset W$ there exists a unique $C^{1}$ solution $x: J \rightarrow$ $\mathscr{D}$ on some open interval $J$ containing the origin of the initial value problem

$$
A(x) \dot{x}=G(x), \quad x(0)=x^{0} .
$$

Moreover, no $C^{1}$ solution of (3.11) exists for $x^{0} \notin W_{1}$.

Definition 3.2 does not rule out the existence of points $x^{0} \in W$ where rank $A\left(x^{0}\right)_{\mid T_{x^{0}} W}<r$, but then $G\left(x^{0}\right) \notin \operatorname{rge} A\left(x^{0}\right)_{\mid T_{x_{0}} W}$, and hence such points do not belong to the set $W_{1}=\pi(T W \cap M)$ (see Remark 3.1). In particular, by Theorem 3.1, no $C^{1}$ solution to the corresponding initial value problem (3.11) exists. Nevertheless, in analogy to Definition 2.3, "one-sided" solutions may well occur at such points:

Definition 3.3. A solution of the initial value problem (3.11) at a point $x^{0} \in W$, $x^{0} \notin \pi(T W \cap M)$, is any continuous function $x: J \rightarrow \mathscr{D}$ defined on an interval of the form $J=[0, T)$ or $J=(-T, 0]$ for some $T>0$ which is of class $C^{1}$ on $J^{0}=J \backslash\{0\}$ and satisfies $x(0)=x^{0}$ and $A(x(t)) \dot{x}(t)=G(x(t))$ for $t \in J^{0}$.

In [12] a precise definition of accessible and inaccessible impasse points of geometrically nonsingular DAEs of index 1 (and higher) is given, where the existence of one-sided solutions can be guaranteed. We shall not repeat this theory here but summarize the main result in the form of the following theorem (see [12, Lemma 5.1 and Theorem 5.1]).

Theorem 3.2. Let (3.1) be a geometrically nonsingular DAE with index 1. The point $x^{0} \in W$ is an accessible or inaccessible impasse point of $(3.1)$ if and only if $y^{0}=\varphi^{-1}\left(x^{0}\right)$ is an accessible or inaccessible standard singular point, respectively, of the reduction (3.9) of (3.1) locally near $x^{0}$. Then, the initial value problem (3.11) has exactly two solutions in the sense of Definition 3.3, both defined either on $J=[0, T)$ or $J=(-T, 0]$ for some $T>0$. Moreover, $\|\dot{x}(t)\|$ tends to infinity as $t$ tends to zero.

As an illustration we consider the first and third examples of [6], which have the form

$$
\left(\begin{array}{lll}
1 & 0 & 0 \\
0 & 1 & 0 \\
0 & 0 & 0
\end{array}\right) \dot{x}=G_{j}(x), \quad x \in R^{3}, j=1,2,
$$

with

$$
G_{1}(x)=\left(\begin{array}{c}
x_{3} \\
x_{3} \\
x_{1}+x_{3}\left(x_{2}+x_{3}^{2}\right)
\end{array}\right), \quad G_{2}(x)=\left(\begin{array}{c}
x_{3}^{2} \\
-x_{3} \\
x_{1}+x_{3}\left(x_{2}+x_{3}^{2}\right)
\end{array}\right) .
$$


Hence, in both examples the conditions of Definition 3.1 are satisfied with $r=2$. Moreover, we have

$$
W=\left\{x \in \mathbb{R}^{3}: x_{1}+x_{3}\left(x_{2}+x_{3}^{2}\right)=0\right\},
$$

and, evidently, the mapping

$$
\varphi: \mathbb{R}^{2} \rightarrow \mathbb{R}^{3}, \quad \varphi(y)=\left(\begin{array}{c}
y_{2}\left(y_{1}+y_{2}^{2}\right) \\
-y_{1} \\
-y_{2}
\end{array}\right), \quad D \varphi(y)=\left(\begin{array}{cc}
y_{2} & y_{1}+3 y_{2}^{2} \\
-1 & 0 \\
0 & -1
\end{array}\right)
$$

is here a global diffeomorphism from $\mathbb{R}^{2}$ onto $W$. Therefore, with the linear isomorphism

$$
L^{0} P^{0}=\left(\begin{array}{lll}
1 & 0 & 0 \\
0 & 1 & 0
\end{array}\right),
$$

the reductions of the two problems have the form

$$
\left(\begin{array}{cc}
y_{2} & y_{1}+3 y_{2}^{2} \\
-1 & 0
\end{array}\right) \dot{y}=H_{j}(y), \quad j=1,2,
$$

where

$$
H_{1}(y)=\left(\begin{array}{l}
-y_{2} \\
-y_{2}
\end{array}\right), \quad H_{2}(y)=\left(\begin{array}{c}
y_{2}^{2} \\
-y_{2}
\end{array}\right) .
$$

Obviously, in both cases, the singular points form the one-dimensional manifold

$$
K=\left\{y \in \mathbb{R}^{2}: y_{1}+3 y_{2}^{2}=0\right\}
$$

and we have $\operatorname{dim} \operatorname{ker} B\left(y^{*}\right)=1$ and $\operatorname{rge} B\left(y^{*}\right)=\operatorname{span}\left(y_{2}^{*},-1\right)^{T}$ for $y^{*} \in K$. Moreover, for $j=1$ we see that $H_{1}\left(y^{*}\right) \notin \operatorname{rge} B\left(y^{*}\right)$ for all $y^{*} \in K$ with $y_{2}^{*} \neq 0,-1$ while for $j=2$ we have $H_{2}\left(y^{*}\right) \in \operatorname{rge} B\left(y^{*}\right)$ for all $y^{*} \in K$. Thus, in the second case, none of the points of $K$ is an impasse point. On the other hand, for $j=1$, a simple calculation with $u=(0,1)^{T} \in \operatorname{ker} B\left(y^{*}\right)$ and $v=\left(1, y_{2}^{*}\right)^{T} \in \operatorname{ker} B\left(y^{*}\right)^{T}$ shows that

$$
\alpha\left(y^{*}\right)(u, v)=-6\left(y_{2}^{*}\right)^{2}\left(y_{2}^{*}+1\right),
$$

whence, all points $y^{*} \in K$ with $y_{2}^{*} \neq 0,-1$ are here impasse points which are accessible for $y_{2}^{*}>-1, y_{2}^{*} \neq 0$ and inaccessible for $y_{2}^{*}<-1$.

Thus, for $j=1,2$ the points $y^{*} \in K, y_{2}^{*}=0,-1$, and for $j=2$ all points $y^{*} \in K$, are higher singularities. In both cases, $y^{*}=0$ is also a stationary point. The differences between these higher singularities and the impasse points in the first example were observed in [6] but explained differently.

\section{Computation of Singular POINTS OF DAEs}

The reduction process for quasi-linear DAEs sketched in the previous section and the resulting theory of impasse points for nonsingular DAEs of index 1 suggests that we may compute such points by applying the augmentation approach of $\S 2$ to the reduced system (3.9). In this section we show that this does indeed lead to an efficient computational algorithm for a variety of singularities including, but not limited to, impasse points.

For simplicity we develop the method only for the following DAE initial value problems occurring frequently in applications:

$$
\left(\begin{array}{c}
A_{1}(x) \\
0
\end{array}\right) \dot{x}=\left(\begin{array}{l}
G_{1}(x) \\
G_{2}(x)
\end{array}\right), \quad x(0)=x^{0} .
$$


Here, $A_{1}: \mathscr{D} \rightarrow \mathscr{L}\left(\mathbb{R}^{n}, \mathbb{R}^{r}\right), G_{1}: \mathscr{D} \rightarrow \mathbb{R}^{r}, G_{2}: \mathscr{D} \rightarrow \mathbb{R}^{\rho}$ are $C^{2}$ maps on some open set $\mathscr{D} \subset \mathbb{R}^{n}$, with $n=r+\rho, \rho>0$, and (4.1) is assumed to be a quasilinear DAE in the sense of Definition 3.1.

The submersion condition for the mapping (3.3) requires that $\operatorname{rank} D G_{2}(x)=$ $\rho$ for $x \in \mathscr{D}$ and hence that the set

$$
N=\left\{x \in \mathscr{D}: G_{2}(x)=0\right\}
$$

is an $r$-dimensional $C^{2}$ submanifold of $\mathscr{D}$.

The manifolds (3.5) and (3.6) are given here by

$$
\begin{gathered}
M=\left\{(x, p) \in \mathscr{D} \times \mathbb{R}^{n}: x \in N, A_{1}(x) p=G_{1}(x)\right\}, \\
W=\left\{x \in N: \operatorname{rank} A_{1}(x)=r\right\},
\end{gathered}
$$

and this allows for some simplification of the reduction process. We denote by $x^{c} \in N$ the points where the local coordinate systems are to be "centered" and computed. More specifically, we follow [14] and use the following tangential local coordinate systems. Let $U^{c}=U\left(x^{c}\right) \in \mathscr{L}\left(\mathbb{R}^{r}, \mathbb{R}^{n}\right)$ define an orthonormal basis of $\operatorname{ker} D G_{2}\left(x^{c}\right)$. Then the implicit function theorem applied to the equation

$$
G_{2}\left(x^{c}+U^{c} y+D G_{2}\left(x^{c}\right)^{T} z\right)=0, \quad y \in \mathbb{R}^{r}, z \in \mathbb{R}^{\rho},
$$

guarantees the existence of open neighborhoods $\mathscr{U}_{c}$ of the origin of $\mathbb{R}^{r}$ and $\mathscr{V}_{c} \subset \mathbb{R}^{n}$ of $x^{c}$ such that for any $y \in \mathscr{U}_{c}$ there exists exactly one solution $z$ of (4.5) with $x^{c}+U^{c} y+D G_{2}\left(x^{c}\right)^{T} z \in \mathscr{V}_{c}$, and that the mapping $\psi: \mathscr{U}_{c} \mapsto \mathbb{R}^{\rho}$, $\psi(y)=z$ is of class $C^{1}$ on $\mathscr{U}_{c}$. Evidently, we have $\psi(0)=0$ and $D \psi(0)=0$, and

$$
\varphi: \mathscr{U}_{c} \mapsto \mathbb{R}^{n}, \quad \varphi(y)=x^{c}+U^{c} y+D G_{2}\left(x^{c}\right)^{T} \psi(y) \forall y \in \mathscr{U}_{c},
$$

is a diffeomorphism from $\mathscr{U}_{c}$ onto $N \cap \mathscr{V}_{c}$. In other words, $\varphi^{-1}$ is a chart of $N$ at $x^{c}$, and we call $\varphi$ a tangential local coordinate map at $x^{c}$.

As in [14], by shrinking if necessary the neighborhoods $\mathscr{U}_{c}$ and $\mathscr{V}_{c}$, we can extend $U^{c}=U\left(x^{c}\right)$ to a moving frame on $\mathscr{V}_{c}$, that is, to a $C^{1}$ mapping $U: \mathscr{V}_{c} \rightarrow \mathscr{L}\left(\mathbb{R}^{r}, \mathbb{R}^{n}\right)$ such that the columns of $U(x)$ form an orthonormal basis of $\operatorname{ker} D G_{2}(x)$ for each $x \in \mathscr{V}_{c}$.

Then, for $y \in \mathscr{U}_{c}$ and $x=\varphi(y)$ we have $(x, p) \in M$ exactly if

$$
B(y) p=H(y), \quad B(y) \equiv A_{1}(\varphi(y)) D \varphi(y), H(y) \equiv G_{1}(\varphi(y)) .
$$

Hence, if $x^{c} \in W$, then necessarily $\operatorname{rank} A_{1}(x)=r$ for $x \in \mathbb{R}^{n}$ in some neighborhood of $x^{c}$ and thus, by restricting again, if needed, the neighborhoods $\mathscr{U}_{c}$ and $\mathscr{V}_{c}$, we find that

$$
B(y) \dot{y}=H(y)
$$

represents for $y \in \mathscr{U}_{c}$ the reduction of (4.1) locally near $x^{c}$.

For the computation we need to be able to evaluate $\varphi(y)$ and $D \varphi(y)$ for $y \in \mathscr{U}_{c}$. There are various possibilities for computing $x=\varphi(y)$ for given $y$. For example, as discussed in [14], we may use at $x=x^{c}$ the QR factorization

$$
D G_{2}(x)^{T}=\left(Q_{1}(x), Q_{2}(x)\right)\left(\begin{array}{c}
R(x) \\
0
\end{array}\right), \quad \operatorname{rge} Q_{2}(x)=\operatorname{ker} D G_{2}(x),
$$


and then set $U^{c}=Q_{2}\left(x^{c}\right)$. Now, for any given $y \in \mathbb{R}^{r}$ with sufficiently small norm, the chord Newton algorithm

Eval- $\varphi$ : Input: $y, R\left(x^{c}\right), Q_{1}\left(x^{c}\right)$;

1. $x=x^{c}+U^{c} y$

2. while 'no convergence'

2.1. solve $R\left(x^{c}\right)^{T} z=G_{2}(x)$ for $z$;

2.2. set $x:=x-Q_{1}\left(x^{c}\right) z$;

3. Output: $x$

converges to $x=\varphi(y) \in N$ and hence implements the tangential coordinate system.

For the computation of $D \varphi(y)$ at any $y \in \mathscr{U}_{c}$ note that

$$
\left(U^{c}\right)^{T} D \varphi(y)=\left(U^{c}\right)^{T} U^{c}+\left(U^{c}\right)^{T} D G_{2}\left(x^{c}\right)^{T} D \psi(y)=\left(U^{c}\right)^{T} U^{c}=I_{r} .
$$

Moreover, since $D G_{2}(\varphi(y)) D \varphi(y)=0$ it follows that $D \varphi(y)=U(\varphi(y)) K(y)$ for some nonsingular $K(y) \in \mathscr{L}\left(\mathbb{R}^{r}\right)$. Together with (4.10), this implies that $K(y)=U(\varphi(y))^{T} D \varphi(y)$ and therefore that

$$
D \varphi(y)=U(\varphi(y))\left[\left(U^{c}\right)^{T} U(\varphi(y))\right]^{-1} .
$$

Clearly, since $U: \mathscr{V}_{c} \rightarrow \mathscr{L}\left(\mathbb{R}^{r}, \mathbb{R}^{n}\right)$ is of class $C^{1}$, the same holds for $D \varphi$. But it turns out that we do not need $U(x)$ to be a $C^{1}$ moving frame on a neighborhood of $x^{c}$ on $N$. In fact, suppose that $\hat{U}(x) \in \mathscr{L}\left(\mathbb{R}^{r}, \mathbb{R}^{\rho}\right)$ represents, for $x=\varphi(y), y \in \mathscr{U}_{c}$, an arbitrary basis matrix of $\operatorname{ker} D G_{2}(x)$ and that $\hat{U}\left(x^{c}\right)=U\left(x^{c}\right)$. Then we have $U(x)=\hat{U}(x) Q(x)$ with some nonsingular $Q(x) \in \mathscr{L}\left(\mathbb{R}^{r}\right)$, and hence

$$
\begin{aligned}
D \varphi(y) & =U(x)\left[\left(U^{c}\right)^{T} U(x)\right]^{-1}=\hat{U}(x) Q(x)\left[\left(U^{c}\right)^{T} \hat{U}(x) Q(x)\right]^{-1} \\
& =\hat{U}(x)\left[\left(U^{c}\right)^{T} \hat{U}(x)\right]^{-1} .
\end{aligned}
$$

While for points $x$ in a neighborhood of a fixed point $x^{c}$ the particular choice of the basis matrix $U(x)$ does not matter, the orientation of $U\left(\tilde{x}^{c}\right) \equiv \tilde{U}^{c}$ does play a role when we move from $x^{c}$ to another point $\tilde{x}^{c}$ where a new local coordinate system is to be centered. The compatibility condition for charts on a $C^{1}$ manifold requires that $\tilde{U}^{c}=U\left(\tilde{x}^{c}\right)$ tends to $U^{c}=U\left(x^{c}\right)$ when $\tilde{x}^{c} \rightarrow x^{c}$ and, in particular, that both bases have the same orientation:

$$
\operatorname{det}\left(\tilde{U}^{c}\right)^{T} U^{c}>0 \text {. }
$$

This will be guaranteed by applying the moving frame algorithm of [14] in the construction of the new basis $\tilde{U}^{c}$.

As indicated, if (4.1) is a nonsingular DAE of index 1 , then one of the augmentation procedures of $\S 2$ will be applied to the reduced ODE (4.8). In practice, it is useful to work with a larger augmentation (2.14) rather than with (2.8) in order for the process to function also near higher-order singularities than just standard impasse points. For this, the augmentation (2.22) with $q=2$ was chosen, for which $v(y)$ and $\gamma(y)$ are easily determined by $(2.23)$.

For the solution of the initial value problem (4.1) with $x^{0} \in N$ the augmentation is constructed at certain (but not necessarily all) the computed points $x^{c}$ along the trajectory where local coordinate systems are centered. Thus at these points the local coordinate map (4.6) is available, and hence the reduced system (4.8) has the form

$$
B^{c} p=H^{c}, \quad B^{c}=A_{1}\left(x^{c}\right) U^{c}, H^{c}=G_{1}\left(x^{c}\right) .
$$


There are various ways for computing suitable vectors $e, c_{1}, c_{2}$ to ensure that the matrix of the augmented system (2.22), that is, here

$$
\left(\begin{array}{ccc}
B^{c} & -H^{c} & e \\
c_{1}^{T} & 0 & 0 \\
c_{2}^{T} & 0 & 0
\end{array}\right)\left(\begin{array}{cc}
v_{1} & v_{2} \\
w_{1} & w_{2} \\
z_{1} & z_{2}
\end{array}\right)=\left(\begin{array}{ll}
0 & 0 \\
1 & 0 \\
0 & 1
\end{array}\right)
$$

is nonsingular. For relatively small dimensions $r$ we may use, for instance, the singular value decomposition

$$
\left\{\begin{array}{l}
V_{L}^{T}\left(B^{c},-H^{c}\right) V_{R}=(\Sigma, 0), \\
V_{L} \in \mathbb{R}^{r \times r}, V_{R} \in \mathbb{R}^{r+1 \times r+1}, \Sigma=\operatorname{diag}\left(\sigma_{1}, \ldots, \sigma_{r}\right), \sigma_{1} \geq \sigma_{2} \geq \cdots \geq \sigma_{r} \geq 0 .
\end{array}\right.
$$

Let $e_{k}^{m}, k=1, \ldots, m$, denote the natural unit basis vectors of $\mathbb{R}^{m}$. Then, with

$$
V_{R} e_{r+1}^{r+1}=\left(\begin{array}{c}
u_{1} \\
\omega_{1}
\end{array}\right), \quad V_{R} e_{r}^{r+1}=\left(\begin{array}{c}
u_{2} \\
\omega_{2}
\end{array}\right),
$$

we choose the augmenting vectors

$$
e= \pm V_{L} e_{r}^{r}, \quad c_{1}=\frac{u_{1}}{\left\|u_{1}\right\|_{2}}, \quad c_{2}=\frac{u_{2}}{\left\|u_{2}\right\|_{2}}
$$

provided that $u_{1}$ and $u_{2}$ are nonzero; otherwise, $V_{R} e_{j}^{r+1}$ for some suitable lower index $j$ is used. The sign of $e$ in (4.14) will be addressed shortly.

Under the assumption that

$$
\operatorname{rank}\left(B^{c},-H^{c}\right) \geq r-1,
$$

the matrix of (4.12) is nonsingular if and only if $\omega_{1}^{2}+\omega_{2}^{2} \neq 1$. In fact, for any null vector $\left(q^{T}, \xi, \eta\right)^{T} \in \mathbb{R}^{r+2}$ of (4.12) we have

$$
\begin{array}{ll}
\sigma_{i} \bar{q}_{i}=0, & i=1, \ldots, r-1, \\
\sigma_{r} \bar{q}_{r} \pm \eta=0 & \text { for }\left(\begin{array}{l}
\bar{q} \\
\bar{\xi}
\end{array}\right)=V_{R}^{T}\left(\begin{array}{l}
q \\
\xi
\end{array}\right) .
\end{array}
$$

Hence, if (4.15) holds, then $\sigma_{i}>0, i=1, \ldots, r-1$, whence $\bar{q}_{i}=0, i=$ $1, \ldots, r-1$, and $q=\bar{\xi} u_{1}+\bar{q}_{r} u_{2}$. Since, by assumption, $c_{1}^{T} q=c_{2}^{T} q=0$, it follows from (4.14) and (4.16) that

$$
\left(\begin{array}{cc}
u_{1}^{T} u_{1} & u_{1}^{T} u_{2} \\
u_{2}^{T} u_{1} & u_{2}^{T} u_{2}
\end{array}\right)\left(\begin{array}{c}
\bar{\xi} \\
\bar{q}_{r}
\end{array}\right)=\left(\begin{array}{c}
u_{1}^{T} q \\
u_{2}^{T} q
\end{array}\right)=0
$$

where, because of the orthonormality of the vectors (4.13) the determinant of the $2 \times 2$ matrix equals $1-\omega_{1}^{2}-\omega_{2}^{2}$. Thus, if this determinant is nonzero, then $\bar{q}_{r}=\bar{\xi}=0$, whence $q=0$ and $\xi=0$, and also $\eta=0$ by (4.16), and the augmented matrix of (4.12) is invertible. Note that (4.15) holds at every regular or algebraic 1-singular point $y^{c}=\varphi^{-1}\left(x^{c}\right)$ of (4.8) (see Proposition 2.2) and hence, in particular, at every impasse point $x^{c}$.

For the choice of the direction of the vector $e$ of (4.14) suppose that $B^{c}$ is nonsingular, that is, that we are not exactly at a singular point of the reduced system. Then a block LU factorization of the matrix of (4.12) shows that

$$
\operatorname{det}\left(\begin{array}{ccc}
B^{c} & -H^{c} & e \\
c_{1}^{T} & 0 & 0 \\
c_{2}^{T} & 0 & 0
\end{array}\right)=\operatorname{det} B^{c} \operatorname{det}\left(c_{1}, c_{2}\right)^{T}\left(B^{c}\right)^{-1}\left(H^{c},-e\right)
$$


The solution of the augmented system (4.12) gives

$$
I_{2}=\left(c_{1}, c_{2}\right)^{T}\left(v_{1}, v_{2}\right)=\left(c_{1}, c_{2}\right)^{T}\left(B^{c}\right)^{-1}\left(H^{c},-e\right)\left(\begin{array}{cc}
w_{1} & w_{2} \\
z_{1} & z_{2}
\end{array}\right) \text {. }
$$

In accordance with (2.23) we compute now

$$
v^{c}=\left(\begin{array}{cc}
w_{1} & w_{2} \\
z_{1} & z_{2}
\end{array}\right)^{-1}\left(\begin{array}{l}
v_{1} \\
v_{2}
\end{array}\right), \quad \gamma^{c}=\operatorname{det}\left(\begin{array}{cc}
w_{1} & w_{2} \\
z_{1} & z_{2}
\end{array}\right),
$$

whence altogether we find that

$$
\operatorname{sign} \operatorname{det}\left(\begin{array}{ccc}
B^{c} & -H^{c} & e \\
c_{1}^{T} & 0 & 0 \\
c_{2}^{T} & 0 & 0
\end{array}\right)=\operatorname{sign} \operatorname{det} B^{c} \operatorname{sign} \operatorname{det} \gamma^{c}
$$

In line with the theory of $\S 2$ we choose the augmenting vector $e$ such that the left side of (4.17) remains positive. In other words, we use the factorization of the matrix of the augmented system (4.12) to monitor the sign of its determinant and replace the computed $z_{1}$ and $z_{2}$ by their negative values when that determinant is negative.

Once the augmentation vectors $e, c_{1}, c_{2}$ have been constructed, the augmented system (2.22) has a nonsingular matrix for any $y \in \mathbb{R}^{r}$ with sufficiently small norm. Hence for these $y$ the right side $v(y)$ of the explicit ODE (2.12) can be computed by (2.23). This evaluation of $v(y)$ and $\gamma(y)$ is implemented as a subroutine of the following form:

Eval-v: Input: $y, \mathrm{QR}$-factors $Q_{1}\left(x^{c}\right), R\left(x^{c}\right)$, augmentation vectors $e$,

$$
c_{1}, c_{2}
$$

1. Use Eval- $\varphi$ to compute $x=\varphi(y)$;

2. Use (4.11) to compute $D \varphi(y)$ and $B(y)=A_{1}(x) D \varphi(y)$;

3. Form the matrix of (2.22) using the vectors $e, c_{1}, c_{2}$;

4. Solve the augmented system (2.22);

5. Use (2.23) to compute $v(y)$ and $\gamma(y)$;

6. Output: $v(y), \gamma(y)$.

This routine allows us to apply one (or several) steps of a standard explicit Runge-Kutta solver, such as RKF-45 or DOPRI-5, for the approximate solution of (2.12). Note that during one (successful) step of such a Runge-Kutta solver, from, say, $y^{0}$ to $y^{1}$, we obtain in the first and last stages from 'Eval- $\mathrm{v}$ ' the values $\gamma=\gamma\left(y^{0}\right)$ and $\gamma=\gamma\left(y^{1}\right)$, respectively.

Altogether, our algorithm for one step along the solution of (4.1) now has the following general form:

SingDAE: Input: Current point $x^{k}$, suggested step $h^{k}$, tolerance $t o l$, minimal step $h_{\text {min }}$;

1. Set $x^{c}=x^{k}$ and compute the QR factorization (4.9) at $x=x^{c}$;

2. With $U^{c}=Q_{2}\left(x^{c}\right)$ evaluate $B^{c}=A_{1}\left(x^{c}\right) U^{c}$;

3. For $B^{c}$ compute the augmentation vectors $e, c_{1}, c_{2}$ in (4.12);

4. Using Eval-v, take a Runge-Kutta step for (2.12) from $y^{0}=0$ with step $h=h^{k}$ to obtain $y^{1}$, a new step $h^{1}$, and $\gamma^{0}=\gamma\left(y^{0}\right), \gamma^{1}=$ $\gamma\left(y^{1}\right)$; 
5. If 'Step not accepted' then

5.1. Replace $h$ by $h / 2$;

5.2. If $|h| \geq h_{\min }$ then go to 4 else error return;

6. Call Eval- $\varphi$ to compute $x^{k+1}=\varphi\left(y^{1}\right) \in N$;

7. Output $h^{k+1}=h^{1}, x^{k+1}$;

8. If ' $\operatorname{sign} \gamma^{0} \neq \operatorname{sign} \gamma^{\prime}$ ' then

singular point passed; if desired, call rootfinder to compute the point.

When $\gamma$ has different sign at two consecutive points $x^{k}$ and $x^{k+1}$, indicating the presence of a singular point, then a rootfinder is applied in step 8 to determine the stepsize $h$ from $x^{k}$, which gives $\gamma\left(y^{1}\right)=0$ and hence which provides the singular point. For this, a simple algorithm of the Dekker-Brent type (see [2]) has been used. If an impasse point has been passed, then $x^{k}$ and $x^{k+1}$ lie on trajectories with opposite orientation. Hence, in order to proceed from $x^{k+1}$, we have to change the sign of the step $h$. At higher-order singular points this may or may not be required.

In steps 1,2 , and 3 of 'SingDAE' a new local coordinate system and new augmentation is computed for each Runge-Kutta step. This has been found adequate in smaller applications. However, for larger problems it is desirable to retain the same coordinate system and augmentation for several Runge-Kutta steps. The reliability of the augmentation can be checked by monitoring the condition of the augmented linear system in 'Eval-v', while the rate of convergence of the chord Newton process of 'Eval- $\varphi$ ' provides a good indicator for the validity of the current local coordinate map. If one of these tests fails, then the output of 'SingDAE' is not accepted and the routine is restarted with a new local coordinate system and augmentation.

There should be no need to enter into the details of such a modification of the process. The approach has similarities with that employed in [8].

\section{NUMERICAL EXAMPLES}

As noted in the introduction, impasse points for DAEs arise frequently in nonlinear circuit problems. As an example, we consider a simple circuit consisting of a nonlinear resistor, linear capacitor, and linear inductor in parallel. The characteristic of the resistor is given by $u=\gamma+i^{2}$, where $i$ and $u$ denote the corresponding branch current and voltage drop, respectively. The example was considered earlier by F. Takens (see [17]), and again in [12], and is modelled by the DAE

$$
\left(\begin{array}{llll}
0 & 0 & 1 & 0 \\
0 & 0 & 0 & 1 \\
0 & 0 & 0 & 0 \\
0 & 0 & 0 & 0
\end{array}\right) \dot{x}=\left(\begin{array}{c}
x_{4} \\
x_{2} \\
x_{1}+x_{2}+x_{3} \\
x_{4}-\gamma-x_{1}^{2}
\end{array}\right)
$$

Here, $x_{j}=i_{j}, j=1,2,3$, are the currents in the three branches, $x_{4}=u$ is the voltage drop, and, for simplicity, the capacitance and inductance were normalized to one. It is readily verified that $(5.1)$ is a DAE on all of $\mathbb{R}^{4}$ with $r=2$ in the sense of Definition 3.1. 
In the notation of (4.1) the constraint manifold (4.2) is given here by

$$
N=\left\{x \in \mathbb{R}^{4}: G_{2}(x)=0\right\}, \quad G_{2}(x)=\left(\begin{array}{c}
x_{1}+x_{2}+x_{3} \\
x_{4}-\gamma-x_{1}^{2}
\end{array}\right) .
$$

In this case we can define the following global coordinate mapping on $N$ :

$$
\Phi: \mathbb{R}^{2} \rightarrow N, \quad \Phi(y)=\left(\begin{array}{c}
y_{1} \\
y_{2} \\
-\left(y_{1}+y_{2}\right) \\
\gamma+y_{1}^{2}
\end{array}\right)
$$

and hence the system

$$
\frac{1}{3}\left(\begin{array}{cc}
-1 & -1 \\
2 y_{1} & 0
\end{array}\right) \dot{y}=\left(\begin{array}{c}
\gamma+y_{1}^{2} \\
y_{2}
\end{array}\right)
$$

constitutes a (global) reduction of (5.1). Clearly, (5.4) is a nonsingular ODE for all $y \in \mathbb{R}^{2}$ with $y_{1} \neq 0$. Moreover, all points of $\left\{y \in \mathbb{R}^{2}: y_{1}=0, y \neq 0\right\}$ are standard singular points of (5.4) while $y=0$ is a geometric singular point. Thus (5.1) has the one-dimensional submanifold

$$
\left\{x \in \mathbb{R}^{4} ; x=(0, \xi,-\xi, \gamma)^{T}, \xi \in \mathbb{R}, \xi \neq 0\right\}
$$

of impasse points, while $x^{*}=(0,0,0, \gamma)^{T}$ is a higher singularity. A closer analysis of the example in [12] shows that the singularity at $x^{*}$ has a different character for the four cases (i) $\gamma>1 / 8$, (ii) $1 / 8 \geq \gamma>0$, (iii) $\gamma=0$, (iv) $\gamma<0$. In particular, except in case (iii), the point $x^{*}$ is a funnel point in the terminology of [17].

Figures 1, 2, and 3 show computed results obtained with the algorithm of $\S 4$ applied to (5.1) for the three values $\gamma=1.0, \quad \gamma=0.0625, \gamma=-1.0$,

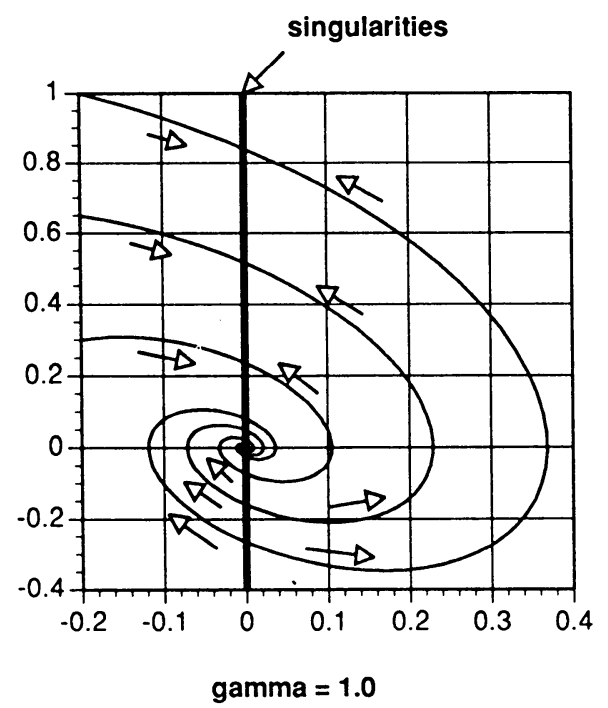

Figure 1 


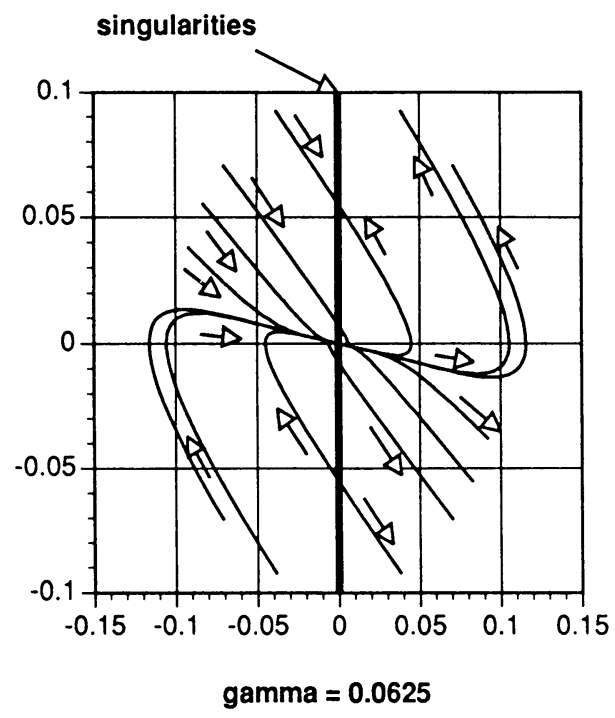

FigURE 2

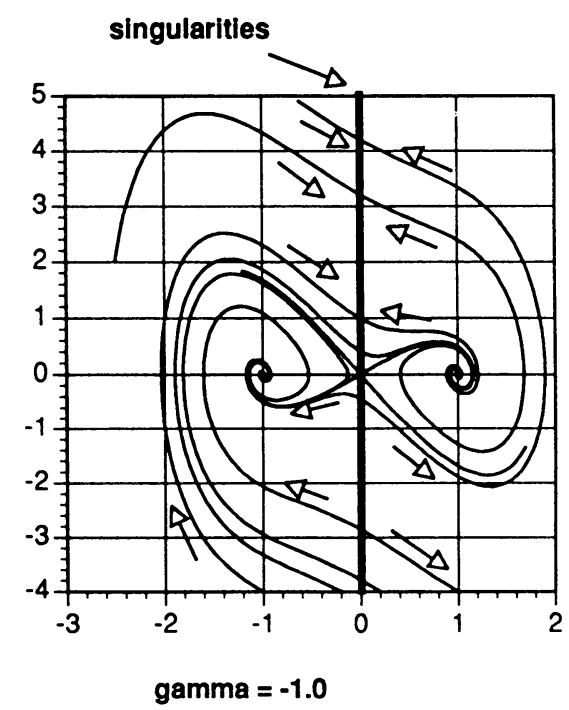

FIGURE 3

respectively. In each case the impasse points $x=(0, \xi,-\xi, \gamma)^{T}$ for $\xi>0$ are accessible while those for $\xi<0$ are inaccessible. Moreover, for $\gamma=-1.0$ the funnel nature of $x^{*}$ is clearly visible. The results certainly show that already very simple circuits may have a relatively complex singularity behavior.

As a second example, we consider a two-phase plug flow problem described by Byrne and Hindmarsh ([4], see also [7]). The equations are given in the form: 


$$
\begin{gathered}
\pi \sqrt{\frac{R}{2 \rho}}(R-y)^{2} \sqrt{-P^{\prime}}\left(2.5 \log \left[\sqrt{\frac{\rho R}{2}} \frac{y}{\mu} \sqrt{-P^{\prime}}-5\right]+10.5\right) \\
-b Q_{c 0}-\frac{P_{0}}{P} Q_{c 0}(1-b)=0 \\
2 \pi \sqrt{\frac{R}{2 \rho}} \sqrt{-P^{\prime}}\left(\left(2.5 R y-1.25 y^{2}\right) \log \left[\sqrt{\frac{\rho R}{2}} \frac{y}{\mu} \sqrt{-P^{\prime}}-5\right]\right. \\
\left.+3 R y-2.125 y^{2}-13.6 R \mu \sqrt{\frac{2}{\rho R}} \frac{1}{\sqrt{-P^{\prime}}}\right)-Q_{a}=0,
\end{gathered}
$$

where $P^{\prime}$ is the time-derivative of $P$. With $x_{1}=P, x_{2}=1 / \sqrt{-P^{\prime}}$, and $x_{3}=y \sqrt{-P^{\prime}}$, the system can be written in the form

$$
\begin{aligned}
& x_{2}^{2} x_{1}^{\prime}+1=0, \\
& x_{1}\left(4.2+\log \left(c_{1} x_{3}-5\right)\right)\left(1-x_{2} x_{3}\right)^{2}-c_{2} x_{2}\left(b x_{1}+c_{4}(1-b)\right)=0, \\
& x_{3}\left(\left(2-x_{2} x_{3}\right) \log \left(c_{1} x_{3}-5\right)+\left(2.4-1.7 x_{2} x_{3}\right)\right)-c_{3}=0,
\end{aligned}
$$

where, after scaling the time by a factor $10^{-7}$, the constants are

$$
c_{1}=\frac{R}{\mu} \sqrt{\frac{\rho R}{2}}, \quad c_{2}=\frac{\rho Q_{c 0}}{2.5 \pi \mu R c_{1}}, \quad c_{3}=\frac{10.88}{c_{1}}+\frac{0.4 \rho Q_{a}}{\pi \mu R c_{1}}, \quad c_{4}=10^{-7} P_{0} .
$$

As in [7], we used the values

$$
\begin{aligned}
R & =45.72, \quad \rho=0.814, \quad \mu=0.098, \quad b=0.345, \\
Q_{c 0} & =1.7153 \times 10^{6}, \quad Q_{a}=3.027 \times 10^{5}, \quad P_{0}=1.378 \times 10^{8},
\end{aligned}
$$

for which

$$
c_{1}=2012.47, \quad c_{2}=19.7157, \quad c_{3}=3.48464, \quad c_{4}=13.78 .
$$

It turns out that the system (5.5) has a singularity at $x^{*}=\left(0,0, x_{3}^{*}\right)^{T}$ for $x_{3}^{*}=0.236849$. More specifically, the system has index one in the sense of Definition 3.2, and for the reduced ODE, $x^{*}$ is an algebraic 1-singular point. But, since the form (2.6) is zero at $x^{*}$, this point is not an impasse point. In fact, $x^{*}$ corresponds to a hysteresis point for stationary equations.

The code was applied to the system (5.5), starting from the point

$$
y^{0}=\left(\begin{array}{lll}
13.78, & 0.42256012,0.24921339
\end{array}\right)^{T}
$$

given in [7]. The resulting trajectory is shown in Figure 4. The singularity $y^{*}$ is reached for $t^{*}=1.0958$, where the derivative $y^{\prime}$ becomes infinite. At the singular point the flow is choked, and hence the part of the trajectory beyond that point is physically meaningless. But it is noteworthy that the code has no difficulty in passing through that point. 


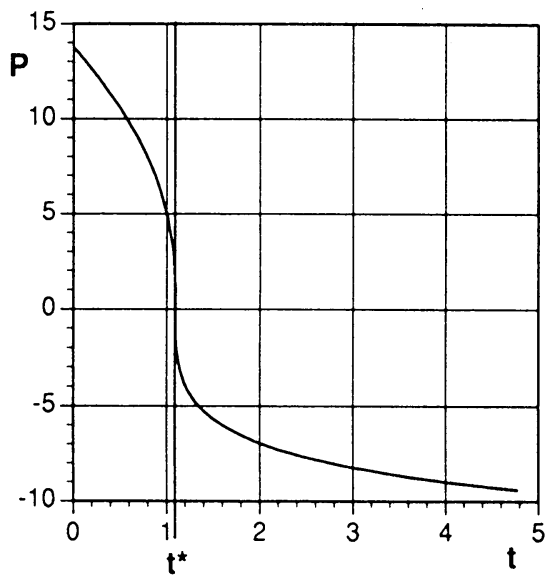

FIGURE 4

\section{BIBLIOGRAPHY}

1. R. Abraham, J. E. Marsden, and T. Ratiu, Manifolds, tensor analysis and applications, 2nd ed., Springer-Verlag, New York, 1988.

2. R. P. Brent, Some efficient algorithms for solving systems of nonlinear equations, SIAM J. Numer. Anal. 10 (1973), 327-344.

3. K. E. Brennan, S. L. Campbell, and L. R. Petzold, Numerical solution of initial-value problems in differential-algebraic equations, North-Holland, New York, 1989.

4. G. D. Byrne and A. C. Hindmarsh, Stiff ODE solvers: A review of current and coming attractions, J. Comput. Phys. 70 (1987), 1-62.

5. L. O. Chua, Introduction to nonlinear networks, McGraw-Hill, New York, 1969.

6. L. O. Chua and A. C. Deng, Impasse points. Part I: Numerical aspects, Internat. J. Circuit Theory Appl. 17 (1989), 213-235.

7. E. Hairer, C. Lubich, and M. Roche, The numerical solution of differential-algebraic systems by Runge-Kutta methods, Lecture Notes in Math., vol. 1409, Springer-Verlag, New York, 1989.

8. F. A. Potra and W. C. Rheinboldt, Differential-geometric techniques for solving differential-algebraic equations, Real-Time Integration Methods for Mechanical System Simulation (E. J. Haug and R. C. Deyo, eds.), Springer-Verlag, New York, 1991, pp. 155-192.

9. P. J. Rabier, Implicit differential equations near a singular point, J. Math. Anal. Appl. 144 (1989), 425-449.

10. P. J. Rabier and W. C. Rheinboldt, A general existence and uniqueness theory for implicit differential-algebraic equations, J. Differential Integral Equations 4 (1991), 563-582.

11. _ A geometric treatment of implicit differential-algebraic equations, Tech. Report TRICMA-162, Inst. Comput. Math. Appl., Univ. of Pittsburgh, June 1991; J. Differential Equations (in press).

12. __ On impasse points of quasilinear differential-algebraic equations, Tech. Report TRICMA-171, Inst. Comput. Math. Appl., Univ. of Pittsburgh, April 1992; J. Math. Anal. Appl. (in press).

13. W. C. Rheinboldt, Numerical analysis of parametrized nonlinear equations, Wiley, New York, 1986.

14. __ On the computation of multi-dimensional solution manifolds of parametrized equations, Numer. Math. 53 (1988), 165-181. 
15. - On the existence and uniqueness of solutions of nonlinear semi-implicit differential algebraic equations, J. Nonlinear Anal. TMA 16 (1991), 647-661.

16. G. W. Stewart, Introduction to matrix computations, Academic Press, New York, 1973.

17. F. Takens, Constrained equations: $A$ study of implicit differential equations and their discontinuous solutions, Lecture Notes in Math., vol. 525, Springer-Verlag, New York, 1976, pp. 143-234.

Department of Mathematics and Statistics, University of Pittsburgh, Pittsburgh, PenNSYlVANIA 15260

E-mail address, P. J. Rabier: rabier@vms.cis.pitt.edu

E-mail address, W. C. Rheinboldt: wrchein@vms.cis.pitt.edu 